New concepts in medicine

\section{The role of cell migration and microchimerism in the induction of tolerance after solid organ transplantation}

\author{
Rahul M Jindal, Amrik Sahota
}

\begin{abstract}
Summary
A new hypothesis has been proposed which states that microchimerism is the basis for the clinical tolerance seen in long-term survivors of solid organ transplants. Efforts to enhance microchimerism include simultaneous infusion of bone marrow of donor origin and transplantation of a solid organ. Studies are in progress to verify the phenomenon of microchimerism and its role in clinical tolerance.
\end{abstract}

Keywords: microchimerism
The ultimate goal in transplantation is to promote the development of donorspecific immunologic unresponsiveness (tolerance), such that immunosuppressants are not required. The development of potent immunosuppressive agents has led to an increased incidence of complications. Therefore, identification of strategies for the induction of tolerance in clinical transplantation is highly desirable.

To achieve this state of tolerance, a variety of techniques such as radiotherapy, polyclonal or monoclonal sera, bone marrow infusion, and nonspecific immunosuppressive agents, have been tried with some success. However, the mechanisms underlying tolerance remain a subject of much debate. A number of theories have been proposed, including clonal deletion of antidonor cytotoxic $\mathrm{T}$-cell precursors in the recipient, peripheral anergy by silencing of the IL-2 (interleukin-2) gene, suppression of alloreactive clonotypes, and clonal paralysis of alloreactive cells by donor 'veto cells'. However, a unified hypothesis has not emerged. ${ }^{1,2}$

The potential of pretransplant blood transfusion in inducing tolerance has been well documented, but this is a nonspecific approach and may result in sensitivity, precluding a later transplant. ${ }^{3}$ Monoclonal antibodies such as antiCD4 have been used to achieve tolerance in a variety of animal models. ${ }^{4}$ Recombinant cytokines such as IL-4 in conjunction with donor-specific transfusion and cyclosporin prolonged survival in the rat heart allograft model. ${ }^{5}$ A particularly novel strategy was to slant the immune response towards Th2 ( $T$ lymphocyte) and away from a Th1 response by the addition of IL-4 in vitro. ${ }^{6}$

The role of 'veto cells' in the induction of tolerance has been the subject of much debate. ${ }^{7}$ Some investigators have suggested that major histocompatibility complex (MHC) class I reactive cells are functionally deleted (vetoed) through interactions with the target cells expressing the specific epitope. However, other investigators found that the induction of tolerance to MHC class II antigens was independent of the veto phenomenon. ${ }^{8}$

\section{Complications of immunosuppressive agents}

It has been known for some time that chronic immunosuppression has numerous disadvantages. It can lead to fatal lymphoproliferative disease, increased incidence of cardiovascular mortality, diabetes mellitus, hyperlipidemia, hypertension, and chronic nephropathy. ${ }^{9}$ Investigators have attempted to reduce the complications of immunosuppressive therapy by lowering or withdrawing steroids, which has often resulted in an increased incidence of rejection episodes. ${ }^{9}$ It has been speculated that the timing of steroid reduction or withdrawal is critical, as late withdrawal has been shown to be better than earlier withdrawal. It has been proposed that those recipients of solid organ transplants who show increased levels of circulating donor cells may be candidates for early steroid reduction, and eventually complete withdrawal of immunosuppressants. ${ }^{10-16}$ Work in our laboratory is directed towards this goal.

\section{Microchimerism: a new concept in graft tolerance}

Indiana University School of Medicine, Indianapolis, Indiana, USA

Department of Surgery

RM Jindal

Department of Medical and Molecular

Genetics

A Sahota

Correspondence to RM Jindal, MD, Department of Surgery, Room 4258, 550-N University Blvd, Indianapolis, IN-46202, USA.

Accepted 17 April 1996
It was recently proposed by Starzl et $a l^{10-12}$ from the University of Pittsburgh that the exchange of migratory leukocytes between the organ transplant and the recipient led to the development of long-term tolerance. This phenomenon has been called microchimerism. The hypothesis is that the presence of donor (microchimeric) cells in recipients of organ transplants correlates with tolerance, and allows for the withdrawal or reduction of immunosuppression.

It was initially shown in female recipients of liver transplants from male cadaveric donors that parts of the macrophage system of the transplanted organ 
were replaced by the cells of the female recipient (identified by the Barr bodies). Since then, there have been a flurry of reports showing that this form of cell migration from the host into the donor organs is a common occurrence. ${ }^{11}$ This unique form of cell migration from the host-to-donor and vice versa (microchimerism) is thought to have important consequences in transplantation. It was proposed that the phenomenon of cell migration could cause accelerated rejection, graft-versus-host-disease (GVHD), or may be the basis of tolerance. On the other hand, it is entirely possible that these cells may simply be innocent bystanders. ${ }^{12}$

The subject of microchimerism and graft tolerance is currently under intense investigation. A number of factors may be involved in this process, such as the quality of immunosuppression, the quantity and quality of immunocytes in the organs, donor-recipient histocompatibility and as yet undefined factors. ${ }^{12}$ It has also been shown that the donor cells departing from the solid organ graft and the recipient cells entering it are dendritic cells. ${ }^{13,14}$ Enhancement of microchimerism, such as by infusion of donor bone marrow, may lead to tolerance, but it could also have deleterious consequences, such as GVHD, or precipitation of acute allograft rejection. ${ }^{15,16}$ The fact that even six-antigenmatched kidneys have only a small advantage, ${ }^{17}$ may perhaps be explained by the phenomenon of microchimerism, whereby tolerance is due to microchimerism, and not to better HLA matching. ${ }^{16}$

A number of factors have been proposed to explain the coexistence of donor and recipient cells in the transplanted patient. These include a variety of cytokines, growth factors, and soluble class I antigens.

\section{Enhancement of chimerism and tolerance by bone marrow infusion}

It has been proposed that microchimerism may be enhanced by the perioperative infusion of bone marrow from the donor. Monaco et al ${ }^{18}$ showed that bone marrow could be a potent tolerogenic agent in recipients of solid organ transplants. What has been described as the 'Monaco model' uses antilymphocyte serum for induction of immunosuppression after infusion of cryopreserved cells after the primary allograft. These findings have been confirmed by others, ${ }^{19,20}$ who have suggested that the induction of tolerance was due to the surviving leukocytes behaving as veto cells.

Clinical trials aimed at enhancing microchimerism by infusion of bone marrow have had mixed results. Workers from the University of Alabama ${ }^{21}$ could demonstrate the presence of mixed allogeneic chimerism in kidney transplant recipients. However, this form of treatment did not result in a significant increase in rejection-free graft survival. Other investigators have failed to detect microchimerism when the liver transplant recipients were given donor bone marrow, despite using a variety of sensitive detection techniques such as polymerase chain reaction. ${ }^{22}$ The infusion of bone marrow did not have any adverse effects on the primary transplanted organ.

Investigators from the University of Pittsburg ${ }^{23}$ have reported the results of clinical trials in which they attempted to augment chimerism by infusion of donor bone marrow. Of the 18 patients reported (10 kidney, 7 liver and 1 heart recipient), they detected the presence of microchimerism in 17 patients, while GVHD was not of clinical significance. The degree of microchimerism was 1000 -fold greater than in control patients, although immunosuppression could not be withdrawn in any patient. They later reported another group of kidney transplant recipients who underwent a combined kidney - bone marrow transplantation. They confirmed their previous results that stable chimerism could be regularly achieved; there was no GVHD, however, the addition of bone marrow did not influence graft function. ${ }^{24}$

\section{Microchimerism in relation to bone marrow transplantation}

GVHD causes serious morbidity and even death in recipients of bone marrow transplantation. Recipients of bone marrow transplantation who suffer from GVHD show the presence of donor type cells in the recipient organs, particularly in the skin, intestinal mucosa, and the bone marrow. It was therefore postulated that the basis of GVHD was the persistence of donor cells in the recipient.

Attempts to enhance microchimerism in organ transplantation may conceivably cause deleterious side-effects. Indeed, some recipients of liver allografts have shown signs and symptoms of GVHD, ${ }^{25,26}$ but is rarely of clinical significance. In clinical trials when simultaneous bone marrow and solid organ transplantation was used to enhance microchimerism, GVHD has not reported to be a problem. ${ }^{21-24}$ 


\section{Techniques for detecting donor-type cells in the transplant recipient \\ - fluorescent in-situ hybridization or polymerase chain reaction to detect the presence of the $\mathrm{Y}$ chromosome in the recipient in case of sex- mismatched transplants \\ - polymerase chain reaction to detect the presence of donor DNA in the recipient by microsatellite analysis or to detect the presence of donor genotypes by SSOP analysis of the HLA-DRB1 region \\ - detection of donor-type MHC in the recipient in tissue sections based on immunohistology using a variety of monoclonal antibodies}

\section{Techniques to detect cells of donor origin in the transplant recipient}

A variety of techniques, based on chromosome analysis, polymerase chain reaction, and immunohistochemistry have been used to detect donor-specific cells in organ transplant recipients. The most widely used techniques are outlined below.

\section{ANALYSIS OF Y CHROMOSOME}

In sex-mismatched transplant cases (male donor, female recipient), DNA from the recipient is analysed by polymerase chain reaction, using $\mathrm{X}$ and $\mathrm{Y}$ chromosome-specific primers. ${ }^{27}$ To minimise contamination, blood and DNA samples are processed by female laboratory staff. Fluorescent in situ hybridisation may also be used for this purpose. Chimerism in male recipients of female donors may be detected by the presence of Barr bodies.

Nagler et $a l^{28}$ were able to detect systemic chimerism in peripheral lymphocytes at a level of $2-5 \%$ following sex-mismatched liver allografts in patients who did not experience rejection, but no donor cells were detected in patients who had clinical episodes of cellular rejection. Ninova et $a l^{29}$ from the Mayo Clinic, also confirmed the utility of the fluorescent in situ hybridisation technique in detecting microchimerism, which was present in $27 \%$ of their sexmismatched liver transplant recipients. The level of donor-type cells was significantly higher in the early posttransplant period; higher level of microchimerism was not, however, associated with an increased incidence of graft rejection.

Work in our laboratory has shown that X-Y chromosome DNA can be readily detected by polymerase chain reaction. We used polymerase chain reaction primers to amplify a portion of the $X-Y$ homologous region, so that two bands were obtained from male DNA and a single band from female DNA. This procedure also acted as an internal control for polymerase chain reaction amplification. Blood samples were obtained from female and male donors and white cells isolated in the usual manner. Different quantities of male cells were added to a fixed quantity $\left(1 \times 10^{5}\right)$ of female cells, such that the percentage of male cells ranged from 0 to $10 \%$. Approximately $1 \%$ of male DNA was readily detectable by gel electrophoresis and ethidium bromide staining of the polymerase chain reaction product. We could increase the sensitivity of this technique by including radioactively labeled dCTP in the reaction mixture.

MICROSATELLITE REPEAT POLYMORPHISMS

Microsatellite sequences (eg, CA repeats) are abundantly interspersed, repetitive DNA elements in the genome of many eukaryotes including humans. These microsatellites are a source of highly informative polymerase chain reaction-based genetic markers that appear to be evenly distributed throughout the human genome. ${ }^{30}$ They have been found to be useful for distinguishing mixed cell populations. The appropriate region is amplified by polymerase chain reaction in the presence of $\mathrm{P}^{32}$-labeled dCTP; the amplified DNA is denatured, and then electrophoresed on a denaturing polyacrylamide gel. The gel is dried and exposed to X-ray film. Each donor and recipient pair is genotyped at several loci before the transplant, and informative microsatellite markers are used in subsequent studies. We are currently using CA repeat markers for mutation studies on chromosome $16 \mathrm{q}$ and have adopted the same polymerase chain reaction assay conditions for microchimerism analysis.

By using the technique of microsatellite sequences, Norris et $a l^{31}$ showed that microchimerism was present in some, but not all, posttransplant recipients. Donor type DNA was found in the peripheral blood, but none was detected from biopsies obtained from the skin and duodenum.

\section{ANALYSIS OF THE HLA-DRB REGION}

Polymerase chain reaction analysis of the HLA-DRB1 region using sequencespecific oligonucleotide probes (SSOP) or sequence-specific primers (SSP) has also been used to detect microchimerism. In SSOP, the region is amplified using generic primers. The polymerase chain reaction products are dot-blotted onto nylon membranes, hybridised with alkaline-phosphatase-labeled SSOPs, and the signal detected by chemiluminescence. ${ }^{32}$ This technique is currently being used in our laboratory for the determination of genotypes at the DRB1 locus. The sensitivity of the technique was determined by mixing experiments where the percentage of donor cells was varied against a fixed population of recipient cells. DNA from these mixed populations was then extracted and genotyped. In SSP, the primers are designed to amplify specific alleles or groups of alleles. 
Investigators from King's College, London, recently reported their results for the detection of donor-specific chimerism in long-term survivors of liver transplants. The recipient and donor HLA-DRB1 region was genotyped by restriction fragment length polymorphism and by SSOP and SSP. They detected donor-specific DRB1 alleles in 39\% patients using nested SSP, while SSOP and one-step SSP could detect only donor-specific chimerism in $22 \%$ and $11 \%$ of the patients tested, respectively. The donor-specific cells were more commonly seen in the skin than in the recipient bone marrow or the blood. They concluded that SSP was the most reliable technique for the detection of donor-specific microchimerism. ${ }^{33}$

\section{THE USE OF MONOCLONAL ANTIBODY}

These are directed to the products of the donor MHC class I or II to stain tissue sections obtained from the transplant recipient, either at biopsy to rule out rejection, or at post-mortem examination. Investigators from the University of Pittsburgh ${ }^{14}$ transplanted liver and heart grafts from hamsters to rats using FK506 (Prograf, tacrolimus) as the immunosuppressive agent. Tissues from graft biopsies were stained with a standard avidin-biotin complex immunoperoxidase technique using specific monoclonal antibodies that recognise class II antigens. From 30 day onwards they demonstrated donor-type dendritic cells in the transplant recipients. Animals that showed higher degrees of cell repopulation had greater survival than those with less repopulation. Similar findings have been noted by other investigators. ${ }^{34}$

\section{QUANTITATIVE ANALYSIS OF MICROCHIMERISM}

The majority of assays for chimerism are qualitative, designed primarily to establish the presence or absence of donor cells. A significant advance would be quantitation of donor cells. Two of the many approaches that may be used to achieve this goal include co-amplification (where an unrelated sequence is used as an internal reference template) and competitive polymerase chain reaction (where two similar templates share the same primer sequences). The products are analysed by densitometry following electrophoresis and autoradiography, and the level of microchimerism is obtained from a standard curve.

\section{Conclusion}

microchimerism is defined as the presence of cells of donor origin in the transplant recipient

- current research is focussed on establishing the relationship between donor-specific microchimerism and clinical tolerance

- if the hypothesis that microchimerism equates with tolerance is valid, then further research to enhance the circulation of cells of donor origin in the recipient will be necessary

- one of the techniques that has been used to enhance microchimerism is to infuse donor bone marrow into the recipient, but such trials to date have been equivocal

Box 3

1 Burlingham WJ, Grailer AP, Fechner Jr JH, et al. Microchimerism linked to cytotoxic T lymphocyte functional unresponsiveness (clonal anergy) in a tolerant renal transplant recipient. Transin a tolerant renal transplant

2 Suberbielle C, Caillat-Zucman S, Legendre C, al. Peripheral microchimerism in long term cadaveric kidney allograft recipients. Lancet 1994; 343: 1468-9.

3 Anderson CB, Brennan D, Keller C, et al. Beneficial effects of donor-specific transfusion on long-term renal allograft function. Transplant Proc 1995; 27: 991 - 4.

4 Bushell A, Pearson TC, Morris PJ, Wood KJ. Donor-recipient microchimerism is not required for tolerance induction following recipient pretreatment with donor-specific transfusion and anti-CD4 antibody. Transplantation 1995; 59: $1367-71$.

5 Levy AE, Alexander JW. Administration of intragraft interleukin-4 prolongs cardiac allograft survival in rats treated with donor-specific transfusion/cyclosporine. Transplantation 1995
59: 405-6.

The concept of donor cell migration into recipient tissues followed by repopulation in the recipient, leading to the induction of tolerance was first put forward by Billingham et al. ${ }^{35}$ This concept has been revived by Starzl in recent years, but it needs to be verified systematically in larger groups of transplant recipients. Recent experimental evidence has suggested that hematopoietic stem-cells residing in the donor liver were responsible for mixed chimerism after liver transplantation. ${ }^{36}$ This is an area of controversy, however, as others have shown that the dendritic cell of donor leukocyte lineage was the basis of microchimerism and tolerance in recipients of solid organ transplants. ${ }^{37}$ The concept of microchimerism has opened new venues of research, which may lead us to the goal of immunosuppression-free transplantation. ${ }^{38}$

We thank the Showalter Trust Fund, University Surgical Associates, Fugisawa, Inc, USA, and the Patel Foundation for supporting our research.

6 Swain SL, Weinberg AD, English M, Huston G. IL-4 directs the development of Th2-like helper effectors. F Immunol 1990; 145: 3796.

7 Miller RG, Phillips RA. Reduction of the in-vitro cytotoxic lymphocyte response produced by invivo exposure to semiallogeneic cells: recruitment or active suppression. F Immunol 1976; 117: 1913.

8 Van Twuyver E, Kast WM, Mooijaart RJD, Melief CJM, De Waal LP. Induction of transplantation tolerance by intravenous injection of allogeneic lymphocytes across an $\mathrm{H}-2$ class II mismatch: different mechanisms operate in tolerization across an $\mathrm{H}-2$ class I vs $\mathrm{H}-2$ class tolerization across an $\mathrm{H}-2$ class I vs $\mathrm{H}-2$

9 Jindal RM. Posttransplant diabetes mellitus - a review. Transplantation 1994; 58: 1289.

10 Starzl TE, Trucco M, Zeevi A, et al. Systemic chimerism in human female recipients of male chimerism in human female recipie
livers. Lancet 1992; 340: 876-7.

11 Starzl TE, Demetris AJ, Trucco $M$, et al. Cell migration and chimerism after whole-organ transplantation: the basis of graft acceptance. Hepatology 1993; 17: 1127-52.
12 Starzl TE. Cell migration and chimerism - a unifying concept in transplantation - with particular reference to HLA matching and tolerance induction. Transplant Proc 1993; 25: 8.

13 Larsen CP, Austyn JM, Morris PJ. The role of graft-derived dendritic leukocytes in the rejection of vascularized organ allografts. Ann Surg 1990; 212: 308-15.

14 Murase N, Starzl TE, Tanabe M, et al. Variable chimerism, graft-versus-host disease, and tolerance after different kinds of cell and whole organ transplantation from Lewis to Brown Norway transplantation from Lewis to Bro
rats. Transplantation 1995; 60: 158 .

15 Starzl TE, Demetris AJ, Trucco $M$, et al. Chimerism and donor-specific non reactivity 27 to 29 years after kidney allotransplantation. Transplantation 1993; 55: 1272-7.

16 Valdivia LA, Demetris AJ, Langer AM, Celli S, Fung JJ, Starzl TE. Dendritic cell replacement in long-surviving liver and cardiac xenografts. Transplantation 1993; 56: 482-4. 
17 Gjertson DW, Terasaki PI, Takamoto S, Mickey $R$. National allocation of cadaveric kidneys by HLA matching: projected effect on outcome and costs. N Engl f Med 1991; 324: 1032-6.

18 Monaco AP, Wood ML, Maki T, Gozzo JJ. Posttransplantation donor-specific bone marrow transfusion in polyclonal antilymphocyte serumtreated recipients: the optimal cellular antigen for induction of unresponsiveness to organ allografts. Transplant Proc 1988; 20: 1207.

19 Thomas JM, Carver FM, Kasten-Jolly J, et al. Further studies of veto activity in Rhesus monkey bone marrow in relation to allograft molerance and chimerism. Transplantation 1989; 47: 66 .

20 Maki T, Gottschalk R, Wood ML, Monaco AP. Specific unresponsiveness to skin allografts in antilymphocyte serum-treated, marrow-injected mice; participation of donor marrow-derived suppressor T cells. f Immunol 1981; 127: 1433.

21 McDaniel DO, Naftilan J, Hulvey $\mathrm{K}$, et al. Peripheral blood chimerism in renal allograft recipients transfused with donor bone marrow. Transplantation 1994; 57: 852-6.

22 Rolles K, Burroughs AK, Davidson BR, et al. Donor-specific bone marrow infusion after orthotopic liver transplantation. Lancet 1994; 343: $263-5$.

23 Fontes P, Rao AS, Demetris AJ, et al. Bone marrow augmentation of donor-cell chimerism marrow augmentation of donor-cell chimerism in kidney, liver, heart, and pancreas

24 Shapiro R, Rao AS, Fontes P, et al. Combined kidney/bone marrow transplantation - evidence of augmentation of chimerism. Transplantation 1995; 59: 306-9.
25 Burdik JF, Vogelsang GB, Smith WJ, et al. Severe graft-versus-host disease in a liver-transplant recipient. $N$ Engl f Med 1988; 318: 689.

26 Schlitt HJ, Raddatz G, Steinhoff G, Wonigeit K, Pichlmayr R. Passenger lymphocytes in human liver allografts and their potential role after transplantation. Transplantation 1993; 56: 951 5.

27 Nakahori $Y$, Hamano $K$, Iwaya $M$, and Nakagome Y. Sex identification by polymerase chain reaction using X-Y homologous primer. $A m \mathfrak{f}$ Med Genet. 1991; 39: 472 .

28 Nagler A, Ilan Y, Ameil A, Eid A, Tur-Kaspa R Detection of systemic chimerism in sex-mismatched liver transplant recipients by fluorescent in situ hybridization (FISH). Hepatology 1993; 18: 339A

29 Ninova $D$, Wiesner $R$, Kondo $M$, Herath J, Jenkins R, Krom RAF. Determination of hematopoietic chimerism in sex-mismatched liver transplant recipients by fluorescence in situ hybridization (abstract P101). International Liver Transplant Congress, 1995.

30 Weber JL, May PE. Abundant class of human DNA polymorphisms which can be typed using the polymerase chain reaction. Am f Hum Genet 1989; 44: 388 .

31 Norris S, Lawlor M, McElwaine S, O'Farrelly C Hegarty I. Assessment of microchimerism following liver transplantation by microsatellite analysis. Hepatology 1995; 22: 209A.
32 Tiercy JM, Morel C, Freidel AC, et al. Selection of unrelated donors for bone marrow transplantation is improved by HIA class II genotyping with oligonucleotide hybridization. Proc Nat Acad Sci USA 1991; 88: 7121 .

33 Thomson L, Donaldson P, Doherty D, Devlin J, Williams R. Detection of donor-specific microchimerism following liver transplantation. Transplantation 1995; 60: 769-70.

34 Yamaguchi Y, Halperin EC, Mori K, et al. Macrophage migration into hepatic xenografts in the hamster-to-rat combination. Transplant Proc 1991; 23: 220 .

35 Billingham RE, Brent L, Medawar PB. "Actively acquired tolerance" of foreign cells. Nature 1953; 172: 603

36 Taniguchi H, Toyoshima T, Fukao K, Nakauchi $H$. Presence of hematopoietic stem cells in the adult liver. Nature Med 1996; 2: 198- 203.

37 Starzl TE, Murase N, Thompson A, Demetris AJ. Liver transplants contribute to their own success. Nature Med 1996; 2: 163-5.

38 Steinman RM, Inaba K, Austyn JM. Donorderived chimerism in recipients of organ transplants. Hepatology 1993; 17: 1153 .

39 Krensky AM, Buelow R, Clayberger C. HLA class I-derived peptides as novel immunosuppressive agents. In: Salamon DR, Sollinger H, eds. Recent developments in transplantation medicine. Glenview: Physicians and Scientists Publishing. Glenview: Physicians; 